\title{
Tape-Drive Based Plasma Mirror
}

\author{
T. Sokollik ${ }^{\mathrm{a}, \mathrm{b}}$, S. Shiraishi ${ }^{\mathrm{a}}$, J. Osterhoff $^{\mathrm{a}}$, E. Evans ${ }^{\mathrm{a}}$, \\ A.J. Gonsalves ${ }^{a}$, K. Nakamura ${ }^{\mathrm{a}}$, J. van Tilborg ${ }^{\mathrm{a}}$, C. Lin $^{\mathrm{a}}$, \\ C. Toth ${ }^{\mathrm{a}}$ and W.P. Leemans ${ }^{\mathrm{a}, \mathrm{b}}$ \\ ${ }^{a}$ Lawrence Berkeley National Laboratory, 1 Cyclotron Road, Berkeley, California 94720, USA \\ ${ }^{b}$ University of California, Berkeley, California 94720, USA
}

\begin{abstract}
We present experimental results on a tape-drive based plasma mirror which could be used for a compact coupling of a laser beam into a staged laser driven electron accelerator. This novel kind of plasma mirror is suitable for high repetition rates and for high number of laser shots.
\end{abstract}

Keywords: plasma mirror, tape drive, staged acceleration.

PACS: 52.38.-r

\section{INTRODUCTION}

In order to design a compact, staged laser plasma based accelerator or collider [1], the coupling of the laser beam into the different stages represents one of the key issues. To limit the spatial foot print and thus to realize a high overall acceleration gradient, a concept has to be found which realizes this in-coupling within a few centimeters (cf. Fig 1). The fluence of the laser pulse several centimeters away from the acceleration stage (focus) exceeds the damage threshold of any available mirror coating. Therefore, in reference [2] a plasma mirror was suggested for this purpose.

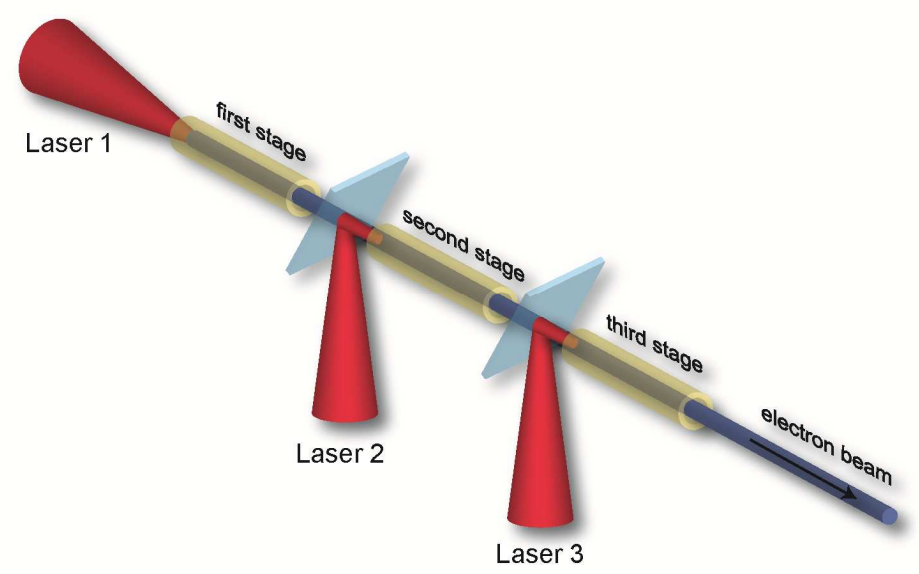

FIGURE 1. Principle of a staged laser driven electron accelerator. Each acceleration stage is driven by a separate laser beam. In order to couple the laser pulses into the stages thin plasma mirrors could be used reducing the distance between the stages.

We present experiments on a tape-drive based plasma mirror which could be used to reflect the focused laser beam into the acceleration stage. Plasma mirrors composed of antireflection coated glass substrates are usually used to improve the temporal laser contrast of laser pulses by several orders of magnitudes [3,4]. This is particularly important for laser interaction with solid matter, such as ion acceleration $[5,6]$ and high harmonic generation on 
surfaces [7]. Therefore, the laser pulse is weekly focused onto a substrate. The main pulse generates a plasma and is reflected at the critical surface, whereas the low intensity pre-pulse (mainly the Amplified Spontaneous Emission pedestal) will be transmitted through the substrate before the mirror has been triggered. Several publications $[3,4]$ demonstrate a conservation of the spatial beam quality and a reflectivity of about $70 \%$. The drawback of this technique is the limited repetition rate since for every shot a fresh surface has to be provided. In the past years several novel approaches for high repetition rate plasma mirrors have been developed $[2,8]$. Nevertheless, for the staged accelerator scheme a second important requirement has to be considered. Since the electron beam has to propagate through the mirror, the thickness of the substrate has to be as thin as possible to reduce the distortion of the electron beam. A tape of only several micrometer thickness can overcome these disadvantages. It can be used with a sufficient repetition rate while it allows the electron beam to propagate through with a minimum of scattering.

\section{EXPERIMENT}

A schematic picture of the tape drive is shown in Fig. 2 (a). Two motors are used to spool the tape with a width of 0.5 inch and a thickness of $25.4 \mu \mathrm{m}$ over several rollers [9]. One of the motors is pulling the tape while the other is used as a tension brake. The surface of the Mylar tape was characterized using an interferometric microscope from the Optical Metrology Laboratory at LBNL. The surface roughness was measured to be about $64.36 \mathrm{~nm}$ (RMS) with a peak to valley value of about $651.6 \mathrm{~nm}$ (see Fig 2 (b)). Additionally, the surface roughness of a Kapton tape and a commercial video tape (VHS) was measured (Fig. 2 (c)). The flattest surface was found for the VHS tape with 14.3 $\mathrm{nm}$ (RMS) and $168.2 \mathrm{~nm}$ peak to valley roughness. Although a higher surface quality would improve the performance of the plasma mirror a Mylar tape was used for the experiments.
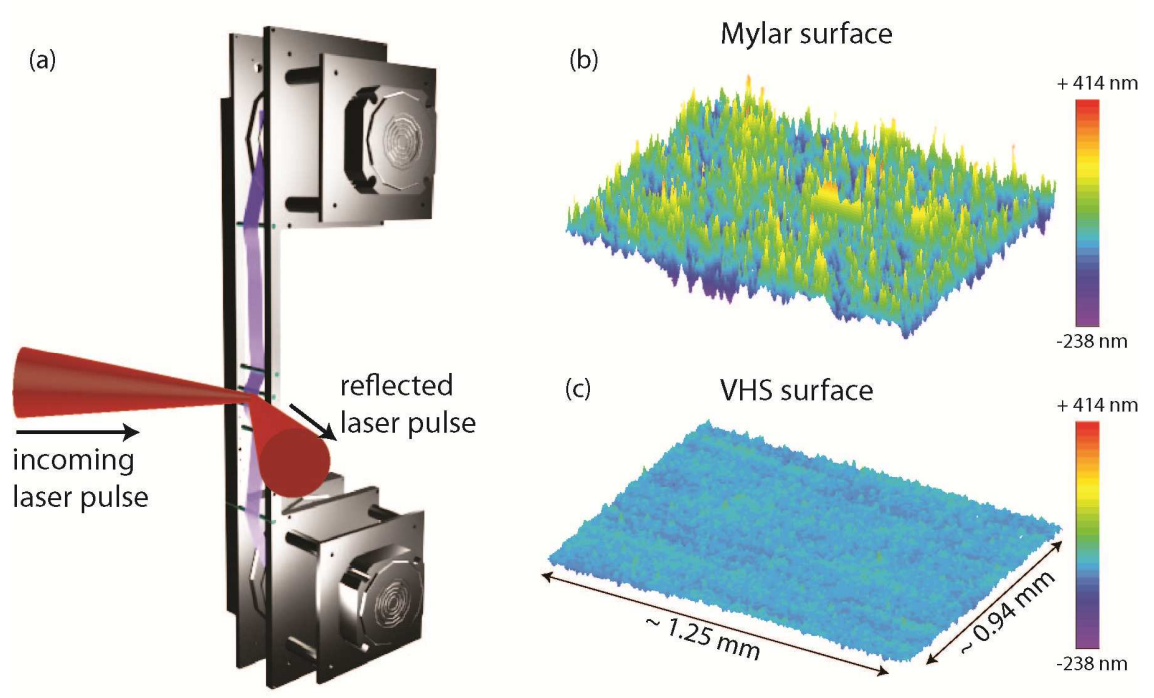

FIGURE 2. (a) Schematic picture of the tape drive. (b) Surface roughness measurement of a Mylar tape. (c) Surface roughness measurement of a VHS tape.

In the experiments a short focal length lens $(15 \mathrm{~cm})$ focused the laser beam with a wavelength of $\lambda=800 \mathrm{~nm}$ to a focal spot of about 6.6 microns (FWHM) on a Mylar tape. Intensities between $10^{12}$ and $10^{17} \mathrm{~W} / \mathrm{cm}^{2}$ were achieved by varying the laser energy of the short pulses ( $42 \mathrm{fs}$ ) between $0.1 \mu \mathrm{J}$ and $5 \mathrm{~mJ}$. To increase the reflectivity of the plasma mirror, s-polarized laser pulses were used. Since the temporal contrast of the laser influences the performance of the plasma mirror, the contrast was measured using a third order autocorrelator (Sequoia). In Fig. 3, a representative correlation trace is shown with a contrast of about $10^{-8}$ up to 5 ps before the main peak. To measure the reflectivity an energy meter and photo diodes were used. The focal distribution of the reflected beam was simultaneously measured by a 12 bit CCD camera. In Fig 4 the energy reflectivity (ratio of the input energy and the reflected energy) is plotted. At low intensities the reflectivity is about $10 \%$, which is consistent for reflection of spolarized light on a Mylar surface. 


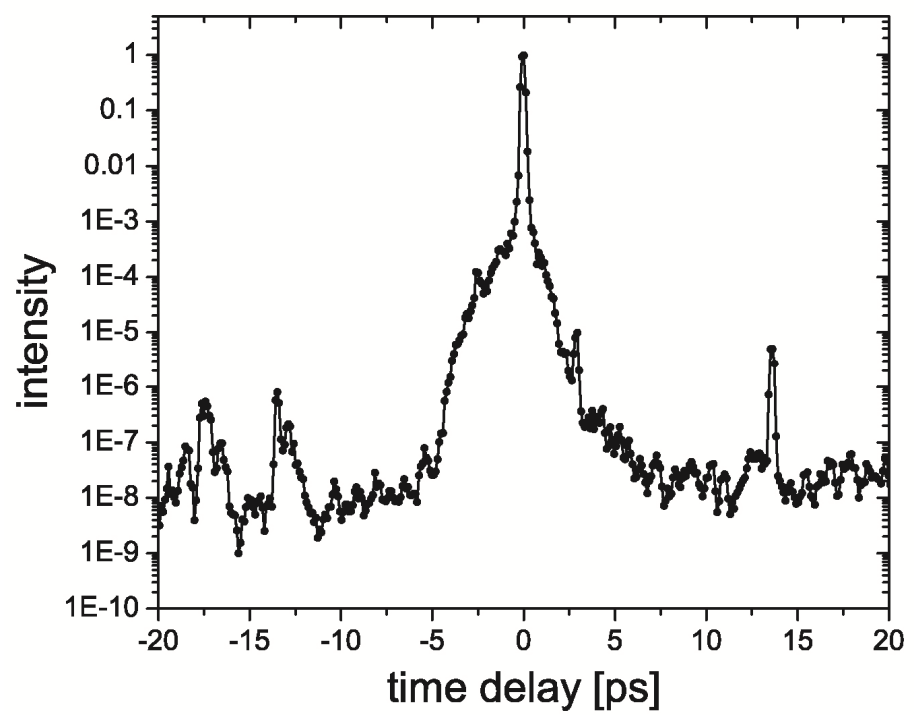

FIGURE 3. $3^{\text {rd }}$ order autocorrelation trace showing a temporal contrast of $10^{-8}$ up to 5 ps before the main pulse.

At higher intensities, at about $10^{13} \mathrm{~W} / \mathrm{cm}^{2}$ the reflectivity starts to increase up to a maximal value of about $78 \%$ at $10^{17} \mathrm{~W} / \mathrm{cm}^{2}$. At these intensities the laser pulse creates a plasma and is reflected at the critical surface. Since the area where a sufficient high intensity is reached to generate an overdense plasma increases for increasing peak intensities, the overall reflectivity increases. Similar reflectivity curves were observed in former experiments [3,4]. Also the beam quality in terms of the Strehl ratio, defined as the ratio of the energy inside the FWHM focus of the measured focal distribution to an ideal Gaussian distribution, is shown in Fig. 4. The Strehl ratio of the incoming beam is about 0.75 . The value of the reflected beam is constant up to an intensity of about $5 \times 10^{15} \mathrm{~W} / \mathrm{cm}^{2}$ and decreases for higher intensities. This can be explained by the expansion of the plasma which can be estimated by the speed of sound $\left(\mathrm{c}_{\mathrm{s}} \approx 7.5 \times 10^{7} \mathrm{~cm} / \mathrm{s}[4]\right)$.

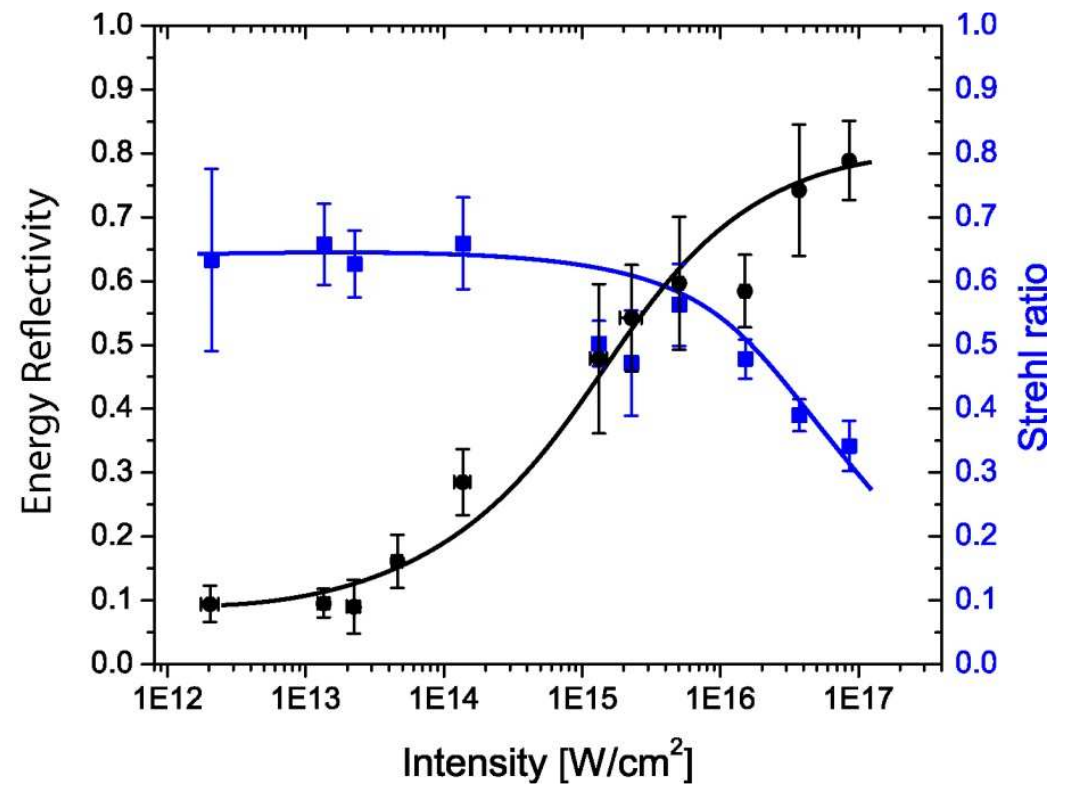

FIGURE 4. Energy reflectivity (black circles) and Strehl ration (blue squares) as a function of the laser intensity. The solid lines are used to guide the eyes. 
If the time between the creation of the plasma and the reflection of the main pulse $(\Delta t)$ is short enough so that no significant expansion of the plasma can occurs $\left(c_{s} \Delta t<\lambda\right)$ the critical surface will be flat and the wave front of the reflected beam will be undistorted. At higher intensities the plasma mirror is triggered earlier in time. If there is enough time for the plasma to expand before the main pulse arrives the reflected wave front will be distorted [4]. Using $\Delta \mathrm{t} \approx 2.5 \mathrm{ps}$ for our contrast parameter and at an intensity of $10^{17} \mathrm{~W} / \mathrm{cm}^{2}$ the plasma expansion can be roughly estimated by $\mathrm{c}_{\mathrm{s}} \Delta \mathrm{t} \approx 1.9 \mu \mathrm{m}$.

Since the tape is moving the stability in terms of beam pointing and reflectivity is important for any application. Therefore, we measured the reflectivity of about 100 shots in two different modes - a single shot mode (moving the tape, tensioning the tape and firing the laser) and a continuous movement of the tape (repetition rate $0.8 \mathrm{~Hz}$ ). The measured reflectively was $(73.5 \pm 6.4) \%$ in case of the single shot mode and $(74.8 \pm 6.6) \%$ for the continuous mode. Simultaneously, the beam pointing was measured by a CCD camera. The laser beam pointing stability before the plasma mirror is about $9.3 \mu \mathrm{rad}$ (RMS). The measured pointing fluctuation after reflection off the plasma mirror is $2.24 \mathrm{mrad}$ and $2.35 \mathrm{mrad}$, respectively (cf. Fig. 5).

(a) single shot mode

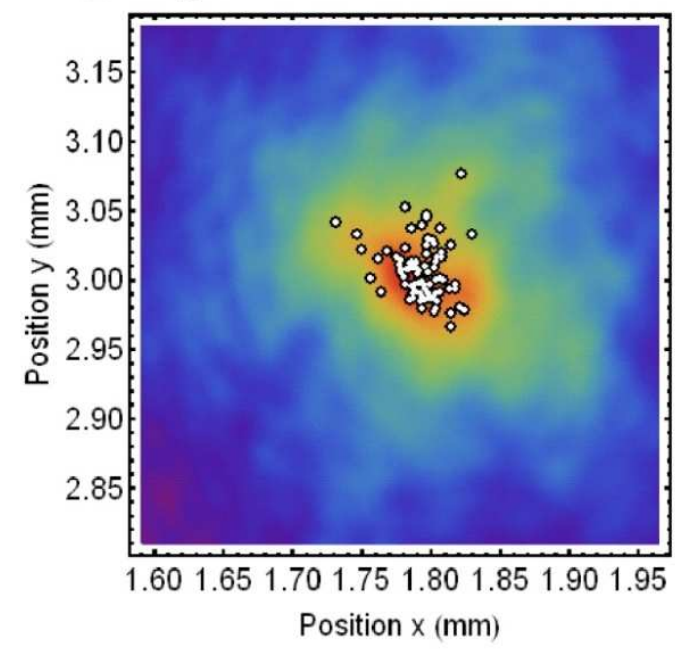

(b) continuous mode

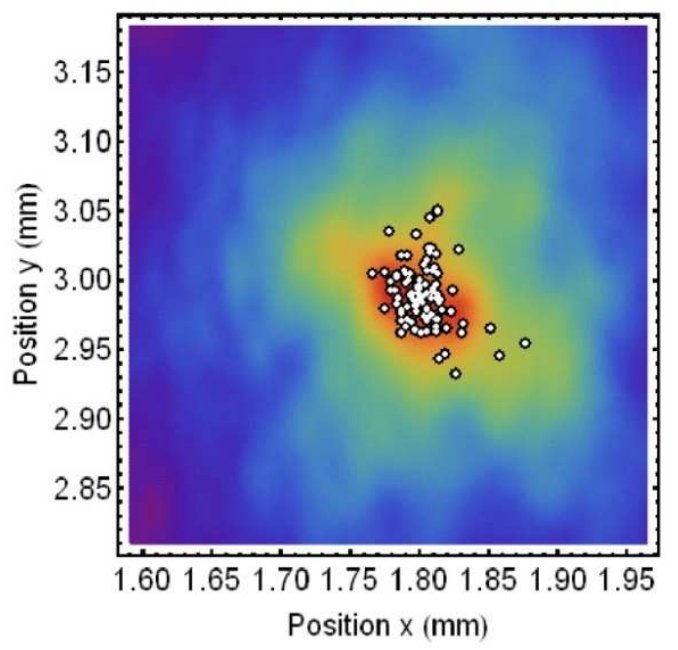

FIGURE 5. Beam pointing measurement for (a) single shot mode and (b) continuous mode. The white dots represent the centroid of the beam for each individual measurement. The background is the sum of all measurements.

The fluctuations in reflectivity and beam pointing could be caused by the surface movement, by fluctuations of the contrast or different surface conditions. Since the laser spot size is on the same order of the surface modulations (cf. Fig. 2 (b)), the latter one is most likely the dominant effect. Using a tape with a better surface quality should reduce the fluctuations in reflectivity as well as in beam pointing and this is being evaluated.

\section{CONCLUSION}

In summary, we presented experimental results using a tape-based plasma mirror. We measured a maximum reflectivity of $78 \%$. Taking the reflected beam quality into account, the optimal intensity range is around $10^{16} \mathrm{~W} / \mathrm{cm}^{2}$ with a reflectivity of about $60 \%-70 \%$. We observed an increased beam pointing variation introduced by the plasma mirror of about $2.3 \mathrm{mrad}$. This value has to be reduced for future applications. This could be reached by using tapes with a higher surface quality, e.g. VHS tapes. The tape could also be used to improve the temporal contrast of the laser pulse. For that purpose p-polarized light would be required to decrease the reflectivity from the tape surface. Additionally, in reference [8] it was proposed to irradiate the mirror at the Brewster angle to improve the contrast enhancement. With these techniques a contrast improvement of roughly 2 orders of magnitude would be feasible. The drawback would be the reduced reflectivity of the main pulse around 50-60\% $[8,10]$. 


\section{ACKNOWLEDGMENTS}

This work was supported by the Director, Office of Science, Office of High Energy Physics, of the U.S. Department of Energy under Contract No. DE-AC02-05CH11231 and the National Science Foundation under Grant No. PHY-0917687.

\section{REFERENCES}

1. W. Leemans and E. Esarey, Physics Today, 62, 44 (2009).

2. D. Panasenko, A. J. Shu, A. Gonsalves, K. Nakamura, N. H. Matlis, C. Toth and W. P. Leemans, J. Appl. Phys., accepted (2010).

3. G. Doumy, F. Quéré, O. Gobert, M. Perdrix, Ph. Martin, P. Audebert, J. C. Gauthier, J.-P. Geindre, and T. Wittmann, Phys. Rev. E 69, 026402 (2004)

4. B. Dromey, S. Kar, M. Zepf, and P. Foster, Rev. Sci. Instrum. 75, 645-648 (2004).

5. A. Henig, S. Steinke, M. Schnürer, T. Sokollik, R. Hörlein, D. Kiefer, D. Jung, J. Schreiber, B. M. Hegelich, X. Q. Yan, J. Meyer-ter-Vehn, T. Tajima, P. V. Nickles, W. Sandner, and D. Habs, Phys. Rev. Lett. 103, 245003 (2009)

6. A. A. Andreev, S. Steinke, T. Sokollik, M. Schnurer, S. Ter Avetisyan, K. Yu Platonov, and P. V. Nickles, Physics of Plasmas, 16, 013103 (2009).

7. C. Thaury, F. Quéré, J.-P. Geindre, A. Levy, T. Ceccotti, P. Monot, M. Bougeard, F. Réau, P. d'Oliveira, P. Audebert, R. Marjoribanks and Ph. Martin, Nature Physics 3, 424 - 429 (2007).

8. Y. Nomura, L. Veisz, K. Schmid, T. Wittmann, J. Wild and F. Krausz, New J. Phys. 9, 9 (2007).

9. T. Plettner, R. L. Byer, E. Colby, B. Cowan, C. M. S. Sears, J. E. Spencer, R. H. Siemann, Phys. Rev. ST Accel Beams 8, 121301 (2005).

10. Y. Cai, W. Wang, C. Xia, J. Liu, L. Liu, C. Wang, Y. Xu, Y. Leng, R. Li, and Z. Xu, Phys. Plasmas 16, 103104 (2009). 
DISCLAIMER: This document was prepared as an account of work sponsored by the United States Government. While this document is believed to contain correct information, neither the United States Government nor any agency thereof, nor The Regents of the University of California, nor any of their employees, makes any warranty, express or implied, or assumes any legal responsibility for the accuracy, completeness, or usefulness of any information, apparatus, product, or process disclosed, or represents that its use would not infringe privately owned rights. Reference herein to any specific commercial product, process, or service by its trade name, trademark, manufacturer, or otherwise, does not necessarily constitute or imply its endorsement, recommendation, or favoring by the United States Government or any agency thereof, or The Regents of the University of California. The views and opinions of authors expressed herein do not necessarily state or reflect those of the United States Government or any agency thereof or The Regents of the University of California. 